\title{
Crystal Morphology Analysis of Piezoelectric Ceramics Using Electron BackScatter Diffraction Method and Its Application to Multiscale Finite Element Analysis*
}

\author{
Yasutomo UETSUJI **, Yu SATOU **, \\ Hideyuki NAGAKURA ***, Hisanao NISHIOKA ***, \\ Hiroyuki KURAMAE **** and Kazuyoshi TSUCHIYA ***** \\ ** Department of Mechanical Engineering, Osaka Institute of Technology \\ 5-16-1 Omiya, Asahi-ku, Osaka, Japan \\ E-mail: uetsuji@med.oit.ac.jp \\ *** Graduate School of Engineering, Osaka Institute of Technology \\ **** Department of Technology Management, Osaka Institute of Technology \\ ***** Department of Precision Engineering, Tokai University \\ 1117 Kitakaname, Hiratsuka, Kanagawa, Japan
}

\begin{abstract}
Microstructural crystal morphology, which affects strongly on macroscopic electromechanical behaviors of polycrystalline piezoelectric ceramics, was analyzed using electron backscatter diffraction method. We coated piezoelectric ceramics with amorphous osmium to defend against electrification caused by electron beam, and measured crystal orientations of $140 \times 120 \mu \mathrm{m}^{2}$ over region at $0.32 \mu \mathrm{m}$ scanning interval. Then the obtained crystal orientations were applied to a multiscale finite element analysis to evaluate the relation with macroscopic mechanical and electrical properties. Especially, we investigated on finite element modeling conditions to sample crystal orientations, and presented a representative volume element of microstructure to compute the macroscopic homogenized properties and the microscopic localized responses.
\end{abstract}

Keywords: Computational Mechanics, Multiscale Finite Element Method, Piezoelectric Ceramics, Electron BackScatter Diffraction, Crystal Morphology

\section{Introduction}

Piezoelectric ceramics, which have been used in actuators or sensors as a component of various electronic and mechanical devices, consist of many crystal grains at a microscopic scale. Since each grain produces strongly anisotropic mechanical and electrical behavior, the macroscopic properties of polycrystalline piezoelectric ceramics have a large dependence of the microscopic crystal morphology. SEM - EBSD technique ${ }^{(1),(2)}$ is well-established and useful to characterize the crystal morphology in polycrystalline materials. Electron beam is irradiated to a specimen in SEM (Scanning Electron Microscope), and then crystal orientations of sub-micron order grains can be analyzed by EBSD (Electron BackScatter Diffraction). The application of SEM·EBSD technique is extended from structural materials ${ }^{(3),(4)}$ to functional ones ${ }^{(5) \sim(7)}$. In our previous studies ${ }^{(7)}$, a crystal morphology analysis was carried out using SEM $\cdot$ EBSD technique for a typical 
piezoelectric ceramics, barium titanate $\left(\mathrm{BaTiO}_{3}\right)$. Because the insulative specimen of piezoelectric ceramics was electrified with electron beam during EBSD measurement, it was however impossible to get high resolution images of crystal orientations such as domain structures.

In this paper, a higher resolution images of crystal morphology is presented for a widely-used piezoelectric ceramics, lead zirconium titanate ( $\left.\mathrm{PZT}: \mathrm{Pb}\left(\mathrm{Zr}_{x}, \mathrm{Ti}_{1-x}\right) \mathrm{O}_{3}\right)$. The insulative specimen of piezoelectric ceramics is coated with a conductive layer of amorphous osmium to remove an electric charge, and crystal orientations in broad region are measured at fine scanning interval. Then the obtained crystal orientations are applied to a multiscale finite element analysis to evaluate the relation with macroscopic mechanical and electrical properties. Especially, a representative volume element of microstructure is investigated to compute the macroscopic homogenized properties and the microscopic localized response.

\section{Crystal Morphology Analysis}

\subsection{Specimen}

A polycrystalline piezoelectric ceramic, PZT (Fuji Ceramics Co., C-91H) was used for the SEM-EBSD specimen. The specimen was a circular disk of $10 \mathrm{~mm}$ diameter and $1 \mathrm{~mm}$ thickness, and it was electrically poled along the thickness direction. The observed surface was mechanically polished for 20 minutes using $3 \mu \mathrm{m}$ diamond particles (Marumoto Struers Co., DP-Spray) with a polishing sheet (Marumoto Struers Co., DP-Mol). And then, it was chemically polished at $\mathrm{pH} 3.5$ for 20 minutes using colloidal particles (Marumoto Struers Co., OP-A) with a polishing sheet (DP-Chem). The surface of the insulative specimen was coated with the electrical conductive and amorphous osmium layer to defend the electrification due to electron beam. We employed an osmium coater (Meiwafosis Co., LTD. Neoc-ST), and set the discharging time in osmium gas to 0.5 seconds. The estimated thickness of osmium layer is $0.5 \mathrm{~nm} / \mathrm{sec}$.

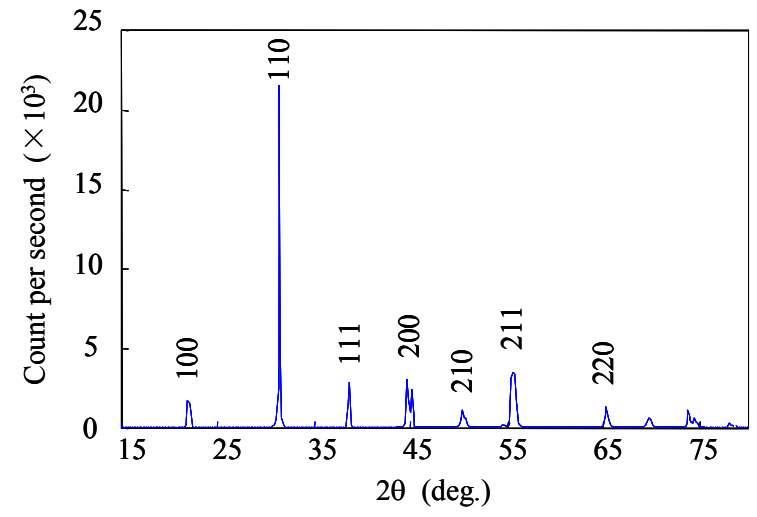

Fig.1 XRD spectrum of PZT piezoelectric ceramics.

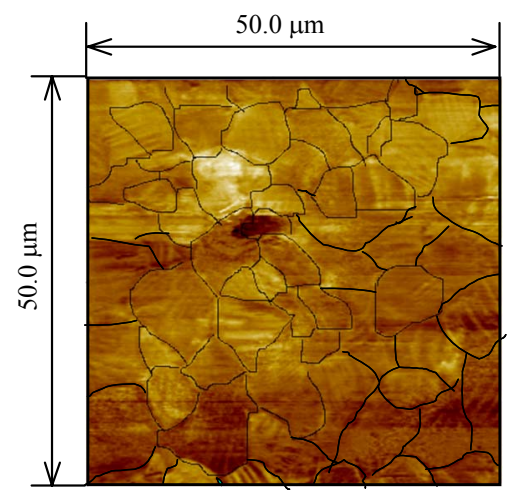

Fig.2 AFM image of the corroded surface of PZT piezoelectric ceramics. 
Prior to the EBSD measurement, the crystal morphology of the specimen was examined by XRD (X-Ray Diffraction) and AFM (Atomic Force Microscope). Figure 1 shows XRD spectrum of PZT piezoelectric ceramics. When the frequency of each orientation was estimated from the intensity of X-ray diffraction, the specimen had the highest orientation to [110] direction. Figure 2 shows AFM image of PZT piezoelectric ceramics. The surface of the specimen was corroded with a hydrofluoric acid to observe domain structures obviously. The number of grains was counted and the grain size was estimated from the maximum dimension of grains. As a result, Fig.2 indicated that the number of grains was 64 and the average of grain size was $7.0 \mu \mathrm{m}$ within $50 \times 50 \mu \mathrm{m}^{2}$ region.

\subsection{EBSD measurement}

Figure 3 shows a crystal morphology image obtained by means of EBSD (Oxford Instruments plc, Link ISIS C.7272) implemented in SEM (JEOL Datum Ltd., JSM-5410). The scanning interval was set to $0.32 \mu \mathrm{m}$ that is smaller than one over twenty of the average grain size. While Fig.3 (a) is the collar map of crystal orientations, (b) is the grayscale map of PQ (Pattern Quality) value defined by Eq.(1).

$$
\mathrm{PQ}=\sum_{i=1}^{3} h_{i} / 3 \sigma_{h}
$$

where $h_{i}$ means the peak height of the Hough transformed $i$-th Kikuchi's band. $\sigma_{h}$ is the standard deviation of Hough transform. The PQ value represents quantitatively the fitting of Kikuchi patterns to the target crystal structure. As shown in Fig.3 (a), several orientations existed in one grain. As all of transgranular orientation gaps were 90 degree, they were considered to be domain structures caused by the domain-switching. The average of PQ values was $45.2 \%$ in Fig. 3 (b), that was $13 \%$ lower than osmium-uncoated specimens.

In order to investigate the representative volume element for the multiscale finite element analysis, the population of crystal orientations requires EBSD data of broad region. If the scanning interval is set to $0.32 \mu \mathrm{m}$, the obtainable maximum region of this SEMEBSD system is $81.9 \times 64.0 \mu \mathrm{m}^{2}$ at once. It may not be enough for the population of crystal orientations. Consequently, crystal orientations in four neighboring regions of $81.9 \times 64.0$ $\mu^{2}$ were measured successively, and their obtained orientations were integrated to keep their real spatial arrangement. As a result, an EBSD data of $141 \times 124 \mu^{2}$ was obtained as shown in Fig.4. Although the details will be discussed in the next section, the representative volume element must be larger than $100 \times 100 \mu \mathrm{m}^{2}$ to make the relative error of the macroscopic homogenized properties $1 \%$ or less. The EBSD data of $141 \times 124 \mu \mathrm{m}^{2}$ in Fig. 4 was enough broad to be a population of crystal orientations.

In order to verify whether the osmium coating is available to defend against electrification, the crystal orientations and the geometric properties of the EBSD data were examined with two measuring methods such as XRD and AFM. First, the crystal

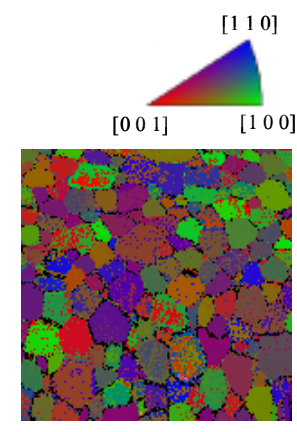

(a) Crystal orientation

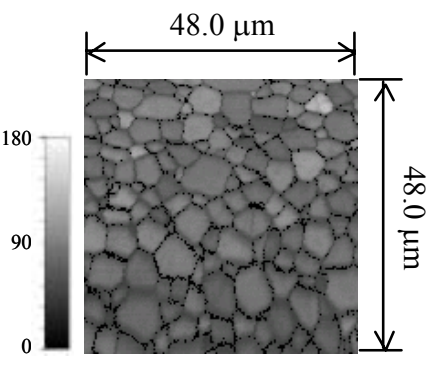

(b) $P Q$ value

Fig.3 Crystal orientation and PQ value distributions in $48 \times 48 \mu \mathrm{m}^{2}$ region obtained by EBSD. 


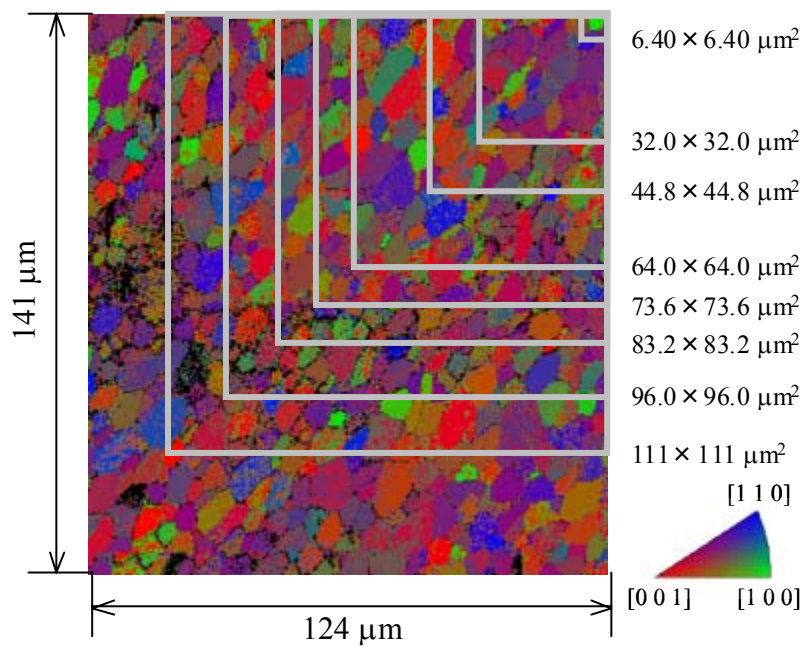

Fig.4 Crystal orientation distribution in $141 \times 124 \mu \mathrm{m}^{2}$ region obtained by EBSD.

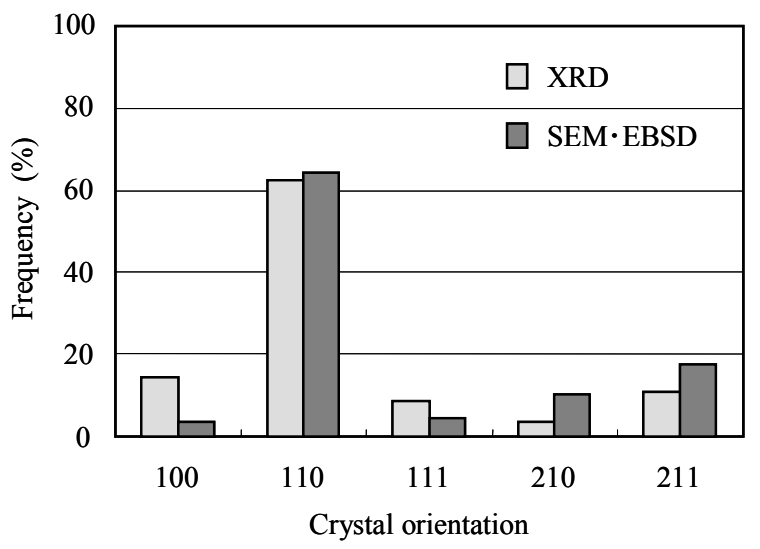

Fig.5 Comparison of the crystal orientation frequency between XRD and EBSD.

Table 1 Comparison of the number and the size of grains between AFM and EBSD.

\begin{tabular}{ccc}
\hline & $\begin{array}{c}\text { Number } \\
\text { of grains }\end{array}$ & $\begin{array}{c}\text { Mean size } \\
\text { of grains }(\mu \mathrm{m})\end{array}$ \\
\hline \multirow{3}{*}{ EBSD } & 64 & 7.25 \\
& 61 & 7.50 \\
& 58 & 6.75 \\
Average & 66 & 6.40 \\
\cline { 2 - 3 } AFM & 62 & 6.98 \\
\hline
\end{tabular}

orientations specified by XRD (Fig.1) were picked out from the EBSD data (Fig.4) and their frequency was calculated. Figure 5 shows the comparison of the crystal orientation frequency between XRD and EBSD. While the XRD data included the overall region of the specimen, the EBSD data was restricted within narrow region of $141 \times 124 \mu^{2}$. However, both data indicated the highest orientation to [110] direction and they had a good correspondence. From this result, the EBSD data of $141 \times 124 \mu \mathrm{m}^{2}$ was considered to satisfy the necessary condition for a population of crystal orientations. Second, four regions of $50.0 \times 50.0 \mu \mathrm{m}^{2}$ were picked out from the EBSD data in Fig.4, and then the number of grains and the grain size were measured to verify the geometric properties. Table 1 shows the number and the mean size of grains of the EBSD data in comparison with the AFM data shown in Fig.2. In case of the AFM data, the number of grains was 64 and the mean size of 
grains was $7.00 \mu \mathrm{m}$. On the other hand, the average number of grains among four regions was 62 and the mean size of grains was $6.98 \mu \mathrm{m}$ in the EBSD data, which had a good correspondence with the AFM data. From the above results, we could consider that the osmium coating of the EBSD specimen unable us to analysis crystal morphology of piezoelectric ceramics without the influence of electrification.

\section{Application to Multiscale Finite Element Analysis}

\subsection{Finite element modeling of microstructural crystal morphology}

The EBSD data of crystal morphology were introduced into microstructural model, and the relation with the macrostructural homogenized properties was investigated through a multiscale finite element analysis based on homogenization theory. As shown in Fig.6, a plate-shaped regular cubic mesh was employed for the finite element model of microstructure. The obtained crystal orientations were inputted into integral points of finite elements by means of three types of model, which had the difference in the frequency and the location of crystal orientations as shown in Table 2. While the EBSD crystal orientations of $111 \times 111 \mu^{2}$ region (Fig.4) was inputted into Model I and II, the XRD crystal orientation frequency was set to Model III. Additionally, crystal orientations were set to integral points which were located at the same coordinates of measuring points in case of Model I. On the other hand, crystal orientations were connected to integral points with a random-number generator in case of Model II and III. Although each crystal grain can be characterized by material constants of a single crystal, any PZT single crystal has not been fabricated and their material constants are unknown. Therefore, the values obtained by the first principles calculation ${ }^{(10)}$ were used for the elastic stiffness constants, the values provided by a polycrystalline PZT ${ }^{(11)}$ were inputted into the dielectric and the piezoelectric stress constants, so that we can evaluate qualitatively the macroscopic material properties outputted from the microscopic crystal morphology.

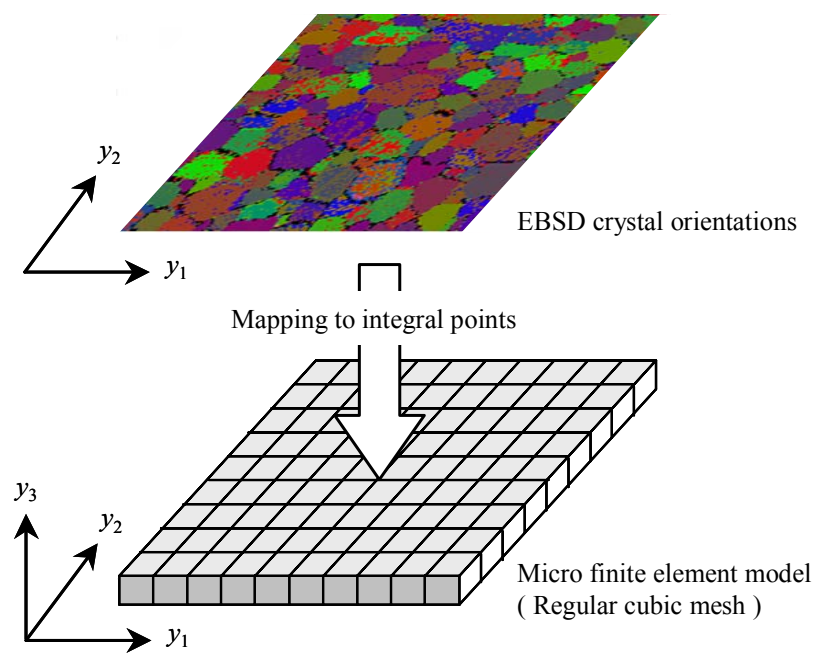

Fig.6 Finite element modeling of microstructural crystal morphology.

Table 2 Three types of finite element model for microstructure.

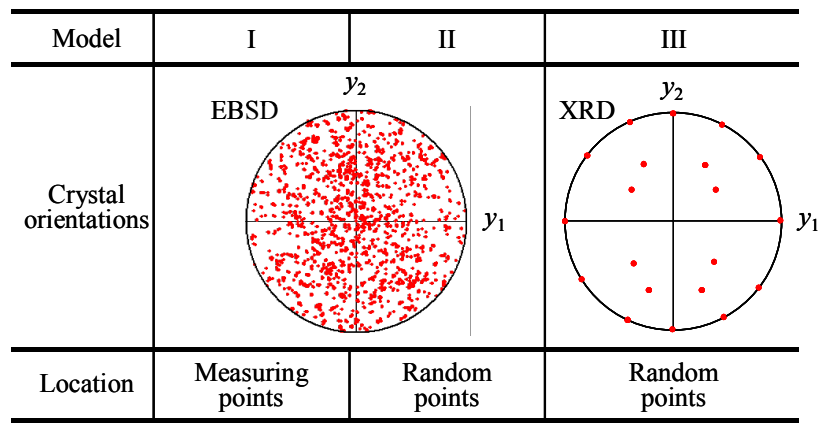


Figure 7 shows the comparison of the macroscopic homogenized elastic compliance constants, and Fig.8 shows the comparison of the macroscopic homogenized dielectric and piezoelectric strain constants among Model I, II and III. The $173 \times 173 \times 1$ divided mesh was employed so as to coordinate the location of the integral points to the EBSD measuring points. Figures 7 and 8 indicated a good accordance of the macroscopic homogenized properties between Model I and II. These computations revealed that the macroscopic homogenized properties had little dependence of spatial distribution of crystal orientations and that they were dominated by the frequency of crystal orientations. On the other hand,

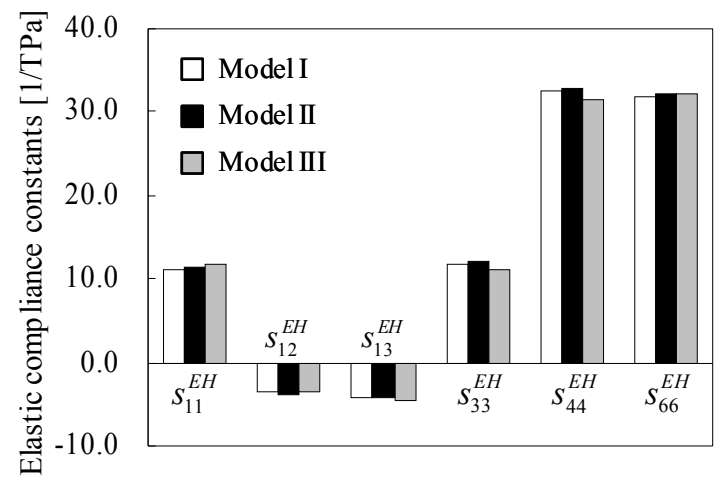

Fig.7 Comparison of macroscopic homogenized elastic compliance constants among Model I, II and III.

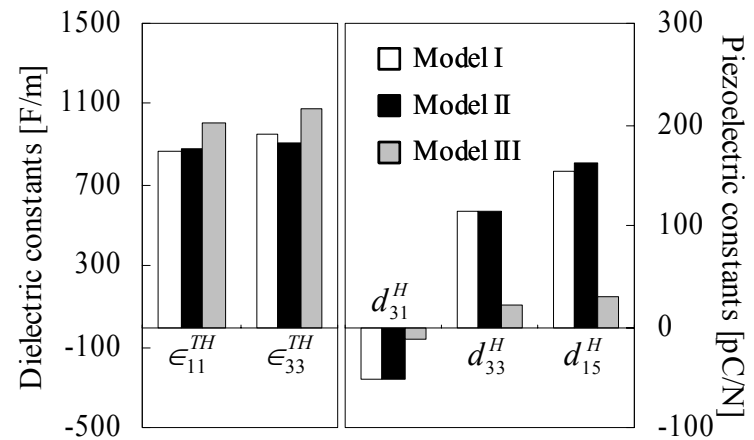

Fig.8 Comparison of macroscopic homogenized dielectric and piezoelectric strain constants among Model I, II and III.

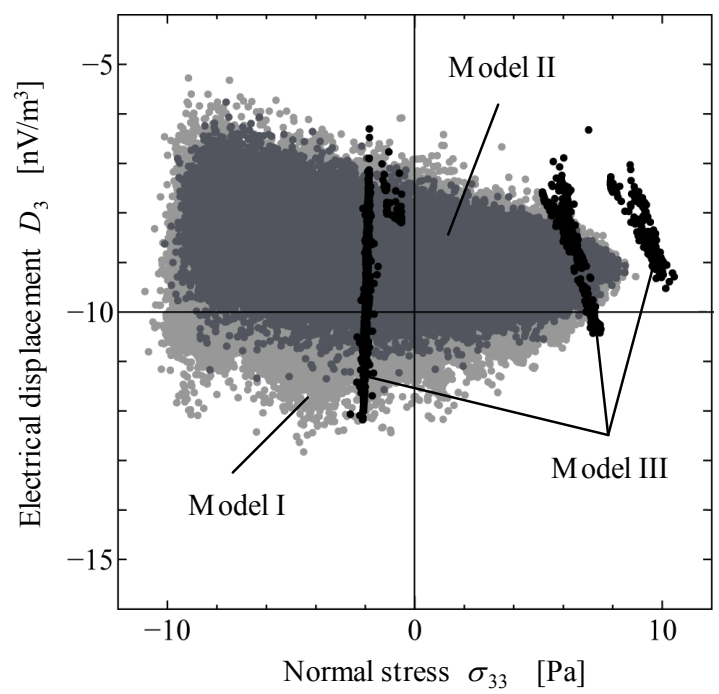

Fig.9 Comparison of microscopic distribution of normal stress $\sigma_{33}^{\text {micro }}$ and electrical displacement $D_{3}^{\text {micro }}$ among Model I, II and III. 
the macroscopic homogenized piezoelectric strain constants provided by Model III made a big difference with Model I and II as shown in Fig.8. Although the frequency of typical orientations corresponded roughly as shown in Fig.5, the XRD data used for Model III could not distinguish finely crystal orientations of low frequency in comparison with the EBSD data inputted into Model I and II. This different frequency of crystal orientations between the XRD and EBSD data made Model III predict lower piezoelectricity of macrostructure.

When an electric field of $1.0 \mathrm{~V} / \mathrm{m}$ was applied to a macrostructure along the $3 \mathrm{rd}$ axis, the microscopic response was analyzed and compared among Model I, II and III. Figure 9 shows the comparison of microstructural distribution of normal stress $\sigma_{33}^{\text {micro }}$ and electrical displacement $D_{3}^{\text {micro }}$ in the 3rd axis. In case of Model III based on the XRD data, the stress and the electrical displacement in the microstructure had a tendency to concentrate on several narrow zones. On the other hand, there was not large difference in case of Model I and II based on the EBSD data. Model II, in which crystal orientations were set randomly to integral points, estimated the stress and the electrical displacement smaller than Model I considering the spatial distribution of measuring points. The aggregation of crystal orientations per grain (or domain) in Model I made the effect of orientation gap stronger, and the higher stress occurred than Model II. Consequently, the consideration of the spatial distribution of crystal orientations, which has little influence on the homogenized properties of macrostructure, is very important for the evaluation of mechanical and electrical behaviors in microstructure.

\subsection{Representative volume element}

The EBSD data within the region from $6.40 \times 6.40 \mu \mathrm{m}^{2}$ to $111 \times 111 \mu \mathrm{m}^{2}$ as shown in Fig. 4 was introduced into microstructure as the same procedure with Model I. And then the homogenized properties of macrostructure and the mechanical and electrical states in microstructure were analyzed to catch a representative volume element of PZT piezoelectric ceramics. The crystal orienting degree $S_{y_{3}}^{c}$ in Eq.(2) was employed for an indicator to estimate the orientation of grains toward an optional direction.

$$
S_{y_{1}{ }^{\prime}}^{c}=\frac{\sum_{i=1}^{n}\left|\boldsymbol{e}_{c}^{i} \cdot \boldsymbol{e}_{y^{\prime}}\right|}{n}
$$

where $\boldsymbol{e}_{c}^{i}$ means the basis vector of $c$ axis of the crystallographic coordinate system in microstructural coordinate system at the $i$-th integral point. $\boldsymbol{e}_{y_{1}}$ is the basis vector of an optional direction $y_{1}$ ' existing in the $y_{1}-y_{2}$ plane. And $n$ is the total number of integral points. The crystal orienting degree $S_{y_{3}}^{c}$ becomes zero when all grains orient to the normal direction of the $y_{1}-y_{2}$ plane and it becomes one when all grains orient to $y_{1}$ ' direction.

Figure 10 shows the crystal orienting degree of the EBSD data within the region from $6.40 \times 6.40 \mu^{2}$ to $111 \times 111 \mu \mathrm{m}^{2}$ in Fig.4. These values were calculated through changing the angle $\theta$ between $y_{1}$ and $y_{1}$ ' from $0^{\circ}$ to $180^{\circ}$. The crystal orienting degree indicated a tendency to converge at a multi-directionally uniform orientation in the $y_{1}-y_{2}$ plane.

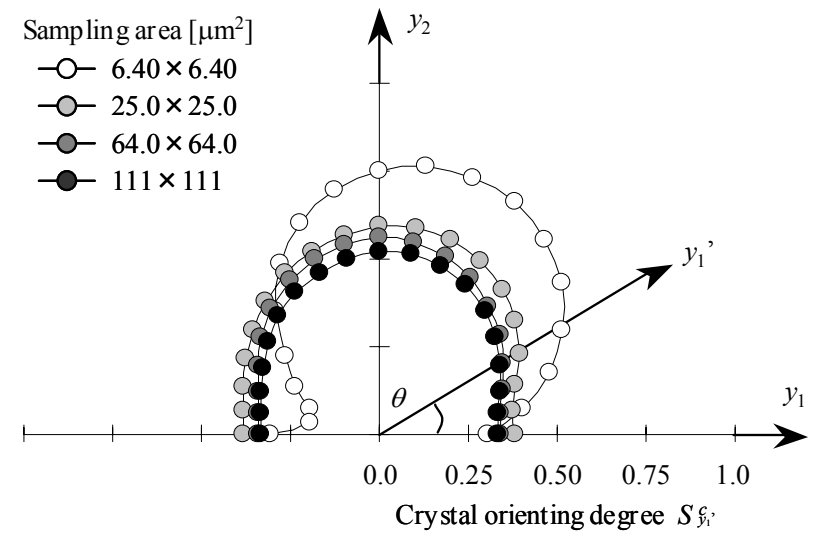

Fig.10 Crystal orienting degree in $y_{1}-y_{2}$ plane of microstructural coordinate system. 
Figure 11 shows the homogenized properties of macrostructure through the multiscale finite element analysis. This figure summarizes (a) nine components of elastic compliance constants, (b) three components of dielectric constants and (c) five components of piezoelectric strain constants, which are including the relative errors averaged per constant. The homogenized material properties of macrostructure converged at a constant value and satisfied the isotropy in the $y_{1}-y_{2}$ plane as the sampling region of the EBSD data increased. These computations indicated that the sampling region must be larger than $100 \times 100 \mu^{2}$ ( 248 grains ) to make the relative error of the homogenized material properties $1 \%$ or less. Next, Fig. 12 shows the mechanical and electrical states in microstructure when an electric field of $1.0 \mathrm{~V} / \mathrm{m}$ was applied to macrostructure along the 3rd axis. This computation was performed for various sampling regions of the EBSD data. The vertical axis indicates (a) the

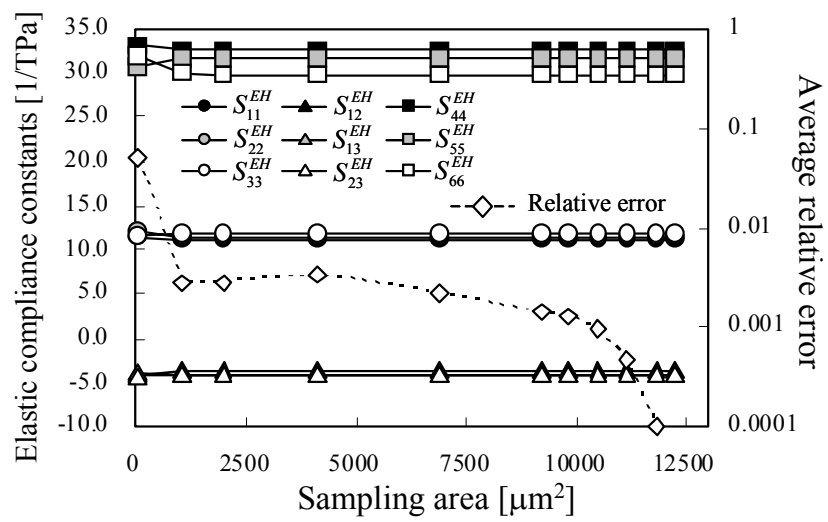

(a) Elastic compliance constants

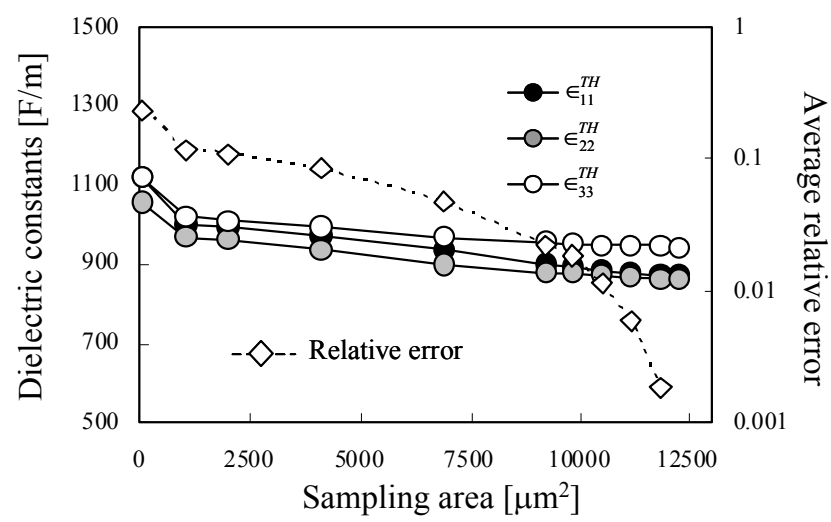

(b) Dielectric constants

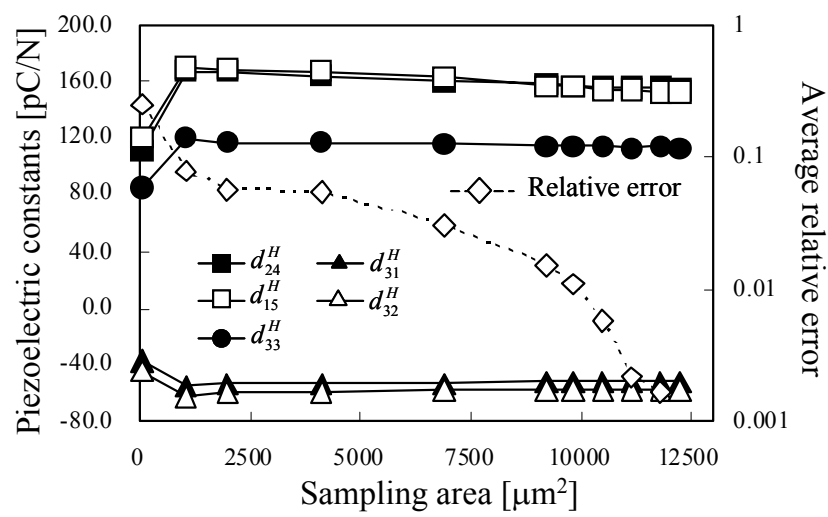

(c) Piezoelectric strain constants

Fig.11 Relation between the macroscopic homogenized properties and the sampling region of crystal orientations. 


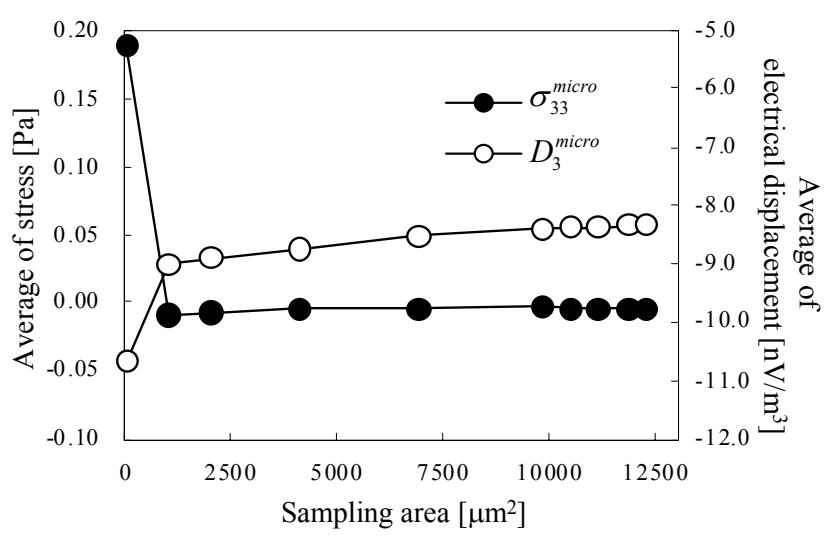

(a) Average

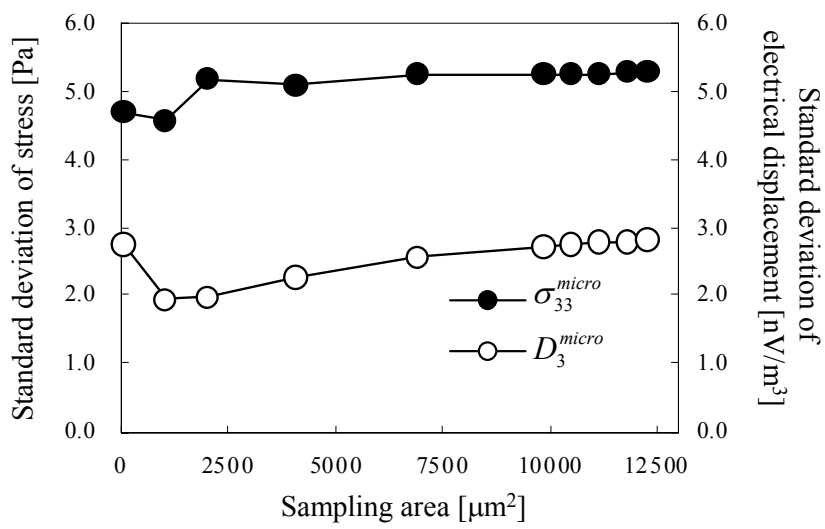

(b) Standard deviation

Fig.12 Relation between the microstructural localized response and the sampling region of crystal orientations.

average and (b) the standard deviation of the normal stress $\sigma_{33}^{\text {micro }}$ and the electrical displacement $D_{3}^{\text {micro }}$ along the $3 \mathrm{rd}$ axis. The average and the standard deviation had the tendency to become a constant value if the sampling region was increasing over $100 \times 100$ $\mu \mathrm{m}^{2}$.

\section{Conclusion}

An osmium coating was employed to fabricate a conductive layer which defends against electrification of the insulative specimen, and then crystal morphology of a conventionally-used PZT piezoelectric ceramics was analyzed using SEM·EBSD method. The obtained EBSD data was verified with the XRD analysis of crystal orientations and the AFM observation of geometric properties. A multiscale finite element analysis was carried out with introduction of the EBSD data into microstructure. As a result, the frequency of crystal orientations was important to estimate the homogenized material properties of macrostructure while the spatial distribution of crystal orientations had a deep influence on the mechanical and electrical states of microstructure. Additionally, it was revealed the representative volume element must be larger than $100 \times 100 \mu \mathrm{m}^{2}$ ( 248 grains ) region to evaluate the macroscopic homogenized properties and the microscopic localized responses.

\section{Acknowledgments}

The authors thank Professor Eiji Nakamachi of Doshisya University for helpful discussions and comments. This study was supported by a Grant-in-Aid for Young Scientists (B) [No. 19760080]. 


\section{References}

(1) Venables, J. and Harland, C., "Electron Back Scattering Patterns - A New Technique for Obtaining Crystallographic Information in the Scanning Electron Microscope", Philosophical Magazine, Vol. 27 (1973), 1193-1200.

(2) Dingley, D. and Randel, V., "Microtexture Determination by Electron Back-Scatter Diffraction", Journal of Material Science, Vol. 27 (1992), 4545-4566.

(3) Kondou, R., Abe, T., Tada, N. and Shimizu, I., "Estimation of Active Slip Plane in Tensile Plastic Deformation of Polycrystalline Copper with EBSD Method", Transaction of the Japan Society of Mechanical Engineers, Vol. 69, No. 681, A (2003), 924-931.

(4) Shimizu, K., Torii, T. and Ochi, H., "Measurement of Crystal Orientation around the Fatigue Crack in Iron Films Using EBSD Method", Journal of the Society of Materials Science Japan, Vol. 53, No. 9, (2004), 987-993.

(5) Yasutomi, Y. and Takigawa, Y., "Evaluation of Crystallographic Orientation Analyses in Ceramics by Electron Back Scattering Patterns (EBSP) Method", Bulletin of the Ceramic Society of Japan, Vol. 37, No. 2 (2002), 84-86.

(6) Khan, A., Carpenter, D. T., Scotch, A. M., Chan, H. M. and Harmer, M. P., "Electron Backscattering Diffraction Analysis of $\mathrm{Pb}\left(\mathrm{Mg}_{1 / 3} \mathrm{Nb}_{2 / 3}\right) \mathrm{O}_{3}-35 \mathrm{~mol}_{0} \mathrm{PbTiO}_{3}$ Single Crystals Grown by Seeded Polycrystal Conversion", Journal of Materials Research, Vol.16, No.3 (2001), 694-700.

(7) Tsunekawa, T., Mizutani, K., Shirakihara, K., Kimachi, H., Tanaka, K. and Andoh, Y., "Microscopic Observation of Domain Switching in Ferroelectric Ceramics under Mechanical Loading", Proceeding of Mechanical Engineering Congress Japan, Vol.1 (2006), 213-214.

(8) Uetsuji, Y., Yoshida, T., Yamakawa, T., Tsuchiya, K., Ueda, S. and Nakamachi, E., "Evaluation of Ferroelectric Properties of Piezoelectric Ceramics and Crystal Orientation Analysis by SEM-EBSD Technique", Transaction of the Japan Society of Mechanical Engineers, Vol. 71, No. 702, A (2005), 241-246.

(9) Uetsuji, Y., Nakamura, Y., Ueda, S. and Nakamachi, E., "Development of Piezo-Electroelastic Finite Element Procedure Based on Crystallographic Homogenization Method", Transaction of the Japan Society of Mechanical Engineers, Vol. 69, No. 679, A (2003), 501-508.

(10) Kitamura, T., Umeno, Y., Shang, F., Shimada, T. and Wakahara, K., "Development of Interatomic Potential for $\mathrm{Pb}(\mathrm{Zr}, \mathrm{Ti})_{3}$ Based on Shell Model", Transaction of the Japan Society of Mechanical Engineers, Vol. 72, No. 718, A (2006), 817-822.

(11) Numamoto, Y. and Ogawa, T., "Dielectric, Piezoelectric and Elastic Constants and their Temperature Dependence in Piezoelectric Single Crystal of PZNT", Memoirs of Shizuoka Institute of Science and Technology, Vol.10, (2002), 55-61. 